\title{
Open hole quasi-static and fatigue characterisation of 3D woven composites
}

\author{
S. Dai ${ }^{\mathrm{a}, *}$, P. R. Cunningham ${ }^{\mathrm{a}}$, S. Marshall ${ }^{\mathrm{b}}$, C. Silva ${ }^{\mathrm{b}}$ \\ ${ }^{a}$ Department of Aeronautical and Automotive Engineering, School of Aeronautical, Automotive, Chemical and \\ Materials Engineering, Loughborough University, LE11 3TU, UK. \\ ${ }^{b}$ M. Wright and Sons Ltd, Quorn Mills, Quorn, Loughborough, LE12 8FZ, UK
}

\begin{abstract}
This paper presents a comprehensive study on the open-hole quasi-static tensile and tension-tension fatigue behaviour of an orthogonal and an angle-interlock 3D woven carbon/epoxy composite. The full-field strain distribution during quasi-static tests was characterised using digital image correlation (DIC), and the fatigue damage behaviour was monitored using an infra-red camera. The notched tensile strength was less than $17 \%$ lower than the un-notched tensile strength and not very sensitive to the notch size. The fatigue specimens were loaded with maximum stress of about $60 \%$ of the ultimate failure stress and no complete fracture occurred after 5,000,000 cycles. The residual fatigue strength was also found to be similar to the quasi-static tensile strength in both weaves. The surface crack initiation and progression during fatigue loading was identified using thermoelastic stress analysis which revealed that the orthogonal weave had larger surface damage area than the angle-interlock weave.
\end{abstract}

Keywords: 3D woven composites; Open-hole tensile tests; Fatigue characterisation; Thermal stress analysis.

\section{Introduction}

The aerospace and automotive industry have shown increasing interest in three-dimensional textile composites due to an excellent damage tolerance and the ability to produce near-net-shape components [1, 2]. Developed from the traditional weaving technology, 3D woven composites provide better through-thickness properties and higher post-impact strength compared to the traditional 2D laminated composites [3, 4]. However, the in-plane quasi-static properties of the $3 \mathrm{D}$ woven composites are generally lower than the 2D laminates due to fibre crimping induced by the interlacing movement during weaving process $[1,5]$, and the fatigue properties of the $3 \mathrm{D}$ woven composites also seem to be lower than the comparable $2 \mathrm{D}$ composites $[2,6,7,8]$. The advantage of $3 \mathrm{D}$ woven composites is that they can be tailored to specific applications where certain mechanical properties (such as impact tolerance) are desired while other properties (such as in-plane tensile strength) are less critical.

Fatigue behaviour and notch sensitivities are two of the main concerns in composite materials since many aerospace applications require mechanical fastening and better fatigue properties. Much

${ }^{*}$ Corresponding author. Email: S.Dai@lboro.ac.uk 
research has concentrated on the un-notched fatigue behaviour of 3D woven composites $[7,6,8,9]$, with less research focused on the notched properties [10, 11]. The tensile fatigue properties of 3D woven composites were found to be better than 2D woven laminates [7], but decrease with increasing content of through-thickness reinforcement $[6,8,9]$. The fatigue sensitivity and the quasi-static tensile strength of the investigated 3D interlock composites was found to be uninfluenced by the presence of a central hole $[10,11,12]$.

Previous work on the fatigue damage progression characterisation for 3D woven composites was generally achieved by pausing the test and examining the specimen with X-ray computed micro-tomography [9] or optical microscopes [9, 10]. Non-destructive testing equipment has been used to continuously monitor the fatigue damage in conventional laminated composites, such as acoustic emission [13, 14, 15], digital image correlation [14, 16], and thermography [14, 13, 17, 15]. However, these technologies have not been used to monitor the in-situ open-hole fatigue damage progression of 3D woven composites to the author's knowledge.

In our previous study [18], six 3D woven composites were tested under tension, compression, and flexure. One orthogonal weave and one angle-interlock weave showed higher tensile properties among all six weaves. Therefore, in this study, the open-hole quasi-static and tension-tension fatigue behaviour of these two weaves are investigated. The full-field strain distribution was obtained via digital image correlation during the quasi-static open-hole tensile tests, and the damage progression was characterised using thermal stress analysis during fatigue tests. The damaged samples were analysed using an optical microscope and post-fatigue tensile strength is also presented.

\section{Materials and manufacture technique}

The 3D woven fabrics were manufactured from a traditional narrow fabric weave loom (Muller-NC2-S) by M.Wright \& Sons Ltd. A 1-by-1 orthogonal weave (W-1) and a through-thickness angle interlock weave (W-3) were produced and Figure 1 shows the idealised weave geometries illustrated using TexGen [19]. Both weaves used 24k IMS5131 yarns as warp tows, $2 \times 6 \mathrm{k}$ HTA40E13 yarns as weft tows. W-1 used 1k Toray T300 as binder tows while W-3 used 3k Tairyfil T33 as binder tows so that both weaves have similar amount of fibres in the through-thickness direction. The woven fabrics were $80 \mathrm{~mm}$ wide, $350 \mathrm{~mm}$ long, and $3 \mathrm{~mm}$ thick, and were placed in a closed mould tool for resin transfer moulding using a Hypaject MK-III RTM system. The resin (Gurit Prime 20LV with slow hardener) was degassed and heated up to $30^{\circ} \mathrm{C}$ and then injected in the preheated and evacuated mould tool. The injection pressure was kept at 1 bar during infusion and was increased to 1.5 bar after the mould was fully filled with resin in order to reduce potential dry spots. The mould tool was then heated up to $50^{\circ} \mathrm{C}$ for 16 hours for curing. The produced composite parts had an overall fibre volume fraction of $49.86 \%$ for $\mathrm{W}-1$ and $46.01 \%$ for $\mathrm{W}-3$, and a warp fibre volume fraction of $27.72 \%$ for $\mathrm{W}-1$ and $25.66 \%$ for $\mathrm{W}-3$. The waviness of the warp tows (defined as tow wave amplitude/unit-cell-length) was also measured on the microscopic samples and was $1.37 \%$ for $\mathrm{W}-1$ and $0.55 \%$ for W-3. Detailed microscopic analysis and mechanical characterisation of the manufactured composites have been reported previously [18]. 


\section{Testing procedure}

\subsection{Open-hole quasi-static tests}

The open-hole tensile tests were carried out using an Instron 6025 testing machine with a $100 \mathrm{kN}$ load cell and a LaVision DIC system to record full-field strain distribution on the surface of the specimens. All of the specimens were loaded at $2 \mathrm{~mm} / \mathrm{min}$ until failure, the load and displacement data were recorded at $2 \mathrm{~Hz}$, and the DIC images were recorded at $4 \mathrm{~Hz}$. According to ASTM D5766 [20], a suggested geometry of the sample is $36 \mathrm{~mm}$ wide by $300 \mathrm{~mm}$ long with a $6 \mathrm{~mm}(1 / 6$ of the width) diameter hole in the centre. However, based on the un-notched tensile strength of these two weaves [18], using a standard sized specimen is likely to result in a failure load (120 kN) higher than the load capacity of the testing machine $(100 \mathrm{kN})$. Therefore the width of the samples was reduced to $25 \mathrm{~mm}$. Previous literature has shown that the 3D woven composites were insensitive to the size of the notch [11], therefore five samples with $4.1 \mathrm{~mm}$ diameter notch (refereed to as "standard" samples) and seven samples with $12.5 \mathrm{~mm}$ notch (refereed to as "enlarged" samples) were tested. The larger notch was used to promote failure in fatigue tests as the available fatigue machine has a load capacity of $25 \mathrm{kN}$. In the absence of standard tests, it is only possible to qualitative comparison with other research findings. The samples were cut from the moulded panel using a diamond saw and the holes were drilled using a silicon carbide drill to minimise damage. Five specimens of each configuration were painted with random white speckle pattern on matt black base and two extra enlarged samples were also tested without the DIC speckle pattern to monitor surface damage evolution.

\subsection{Open-hole fatigue tests}

According to thermoelastic theory, the temperature of a material changes when the material changes volume due to mechanical work, and the temperature change can be related to the stress change in an adiabatic environment using Equation 1 [21]. Generally if the fatigue loading frequency is higher than $5 \mathrm{~Hz}$, the loading process can be treated as adiabatic. Therefore the temperature difference between the peak and valley of the cyclic load can be related to the stress change, and the development of the stresses during the fatigue loading can be treated as an indicator of damage development.

$$
\Delta T=-\frac{T}{\rho C_{p}}\left(\alpha_{11} \Delta \sigma_{1}+\alpha_{22} \Delta \sigma_{2}\right)
$$

where $\Delta T(K)$ is the temperature oscillation during a fatigue cycle on the surface of the specimen, $T(K)$ is the absolute temperature on the surface of the specimen, $\rho\left(\mathrm{kg} / \mathrm{m}^{3}\right)$ is the density, $C_{p}(J / k g K)$ is the specific heat capacity at constant pressure, $\alpha_{11}$ and $\alpha_{22}\left(K^{-1}\right)$ are the surface coefficients of thermal expansion (CTE) in 1 (warp) and 2 (weft) directions, and $\Delta \sigma_{1}$ and $\Delta \sigma_{2}$ are the amplitudes of the principal stresses at the surface [17, 21].

The enlarged-notched samples were used in the fatigue tests so that the maximum fatigue stress was high enough (typically 50-70\% of the static strength) to induce damage to the specimen without loading for a high number of cycles. An Instron 8872 servo-hydraulic fatigue testing machine with a $25 \mathrm{kN}$ load cell was used to apply a sine-wave cyclic load with a mean load of $20 \mathrm{kN}$ and an amplitude of $8 \mathrm{kN}$, which resulted in the peak stress being about $63 \%$ and $61 \%$ of the static open-hole tensile strength of W-1 and W-3 respectively. A loading frequency of $20 \mathrm{~Hz}$ was used to minimise 
non-adiabatic effect and reduce testing time [22, 23]. One surface of the sample was painted in flat matt black to provide a surface with uniform emissivity of about 0.92 [24]. A FLIR Silver 450M infra-red camera was used to record a 2 second video of the surface temperature at 383 frames/second every 10 minutes during the fatigue loading. The first video was recorded after the first 500 cycles when the temperature of the sample had stabilised. The cyclic load signal from Instron 8872 was used as a lock-in signal for the thermal images in order to synchronise the thermal images with the peaks and valleys of cyclic loading. Six samples of each weave were tested for different durations to exam the damage progression at different cycles: two samples for 100,000 cycles, two samples for 500,000 cycles, one sample for 1,000,000 cycles, and one sample for 5,000,000 cycles.

\section{Results and discussion}

\subsection{Open-hole quasi-static tests \\ 4.1.1. Notch strength}

Table 1 presents the averaged open-hole tensile strength with two types of notches and the un-notched tensile strength which was previously published in [18]. The presented notch strength is the net stress calculated based on the cross-sectional area that considering the hole. It can be seen that in both weaves, the enlarged samples had lower notch strength than the standard samples but less than $7 \%$ different. Similar results on other 3D woven orthogonal samples with $4.1 \mathrm{~mm}$ and 11 mm hole have been reported [11]. Although W-3 has lower un-notched tensile strength than W-1, its notched strength was higher than W-1 and showed less degradation from the un-notched tensile strength. Both W-1 samples showed about $18 \%$ reduction from the un-notched strength, while both W-3 samples showed less than $10 \%$ reduction and the standard W-3 samples showed less than $1 \%$ reduction from the un-notched strength. The broadly distributed geometrical flaws and coarse yarns caused debonding and reduced stress concentration around sites of failure, therefore resulted in a lower strength decrease $[11,12]$. These results further confirmed that the stress concentration induced by a circular notch has little influence on the quasi-static tensile strength on 3D woven composites $[10,11,12]$.

\subsubsection{DIC strain distribution}

Figure 2 and 3 show the longitudinal strain distribution of two typical W-1 samples at different stress levels along with the image of the weave pattern. Since the fibre structure of Weave- 1 was highly orthotropic (only contain fibres in 0 and 90 directions) and inhomogeneous (macroscopically distinctive resin rich regions), the strain distribution was not even and showed a strong correlation with the weave structure. The surface weave pattern was extracted and imposed on the DIC maps to emphasis the relationship between the weave pattern and strain distribution. The surface of the sample was dominated by weft tows which were perpendicular to the loading direction. Therefore a simple iso-stress assumption can be used to estimate the surface strain distribution: the region with lower stiffness will exhibit higher strain. As can be seen from Figure 2 (b) and Figure 3 (b) the resin rich channels exhibited higher strain and the centre of the weft flow region (weft region between two adjacent binder) also showed higher strain than the binder tow regions. The local longitudinal stiffness on the surface of the sample was distributed with the resin rich channels being the lowest, surface weft tow being the intermediate, and the binder region being the highest, which resulted in 
this strain distribution. Similar strain patterns have been reported on different types of 3D woven composites [5, 25]. The strain distribution around the notch exhibited a pattern similar to the typical "butterfly" pattern in an isotropic sample. Since the resin rich channels and surface weft tow regions gave rise to strain concentration, the higher strain regions near the notch further extended to these less stiff regions, as highlighted in the black ellipses in Figure 2 (b). At $1182 \mathrm{MPa}$ (the last DIC image before fracture), the influence of the resin channels on the strain distribution was less obvious as at $340 \mathrm{MPa}$, because surface cracks (visually observed) in the notch region and increased the apparent strain in this area. Therefore the high strain concentration areas near the notch in the strain maps (c) in Figure 2-5 were caused by surface cracks. The higher strain region at the upper right conner highlighted in red ellipse in Figure 2 (c) was caused by a local manufacturing defect at the back of the sample, which later developed in to a secondary fracture site. This manufacturing defect did not affect the ultimate tensile strength of this sample since it was the warp tows in the notched region that determined the tensile strength. Similar strain distribution was found in the enlarged samples with higher strain concentrated in the resin rich channels around the notch as can be seen in Figure 3 (b). The strain concentration region near the notch also shifted at higher stress level in Figure 3 (e) due to a visible surface crack in these regions.

The longitudinal strain distribution maps of the W-3 samples are shown in Figure 4 and 5 . The strain distribution of both standard and enlarged samples did not exhibit pronounced features of the weave pattern, because W-3 had smaller surface resin rich regions and was more homogeneous than $\mathrm{W}-1$, which resulted in a more even strain distribution. The resin rich regions around the binding points exhibited higher strain concentration at a later stage of loading as shown in the red ellipse in Figure 4 (c). Surface cracks also developed near the hole and caused the higher strain region shifting to the diagonal direction.

\subsubsection{Failure analysis}

The fractured notched samples of both W-1 and W-3 showed similar failure modes as the un-notched samples, such as warp tow fracture, debonding, and matrix cracking. The damage process during open-hole tests on two enlarged samples were recorded as shown in Figure 6 and 7 .

Figure 6 plots the load-displacement curve of an enlarged W-1 sample with images showing the damage state at different load levels. It can be seen that visible longitudinal cracks occurred along the edge of the hole, which was caused by warp tow straightening and debonding near the hole due to stress concentration in this region. Similar debonding phenomena has been reported previously, and was inferred to be the cause of stress relaxation and therefore the lower notch sensitivity $[10,11]$. The transverse matrix cracks initiated in the resin rich channels where strain concentration existed and propagated along the channel across the width of the specimen. The transverse fracture caused further debonding between the warp and weft tows and eventually resulted in the warp tow and binder tow fracture. The load-displacement curve showed that the sample maintained linear response until about $80 \%$ of the maximum load even after these surface damage occurred. Based on the load-displacement data, the stiffness of this sample has dropped for about $14 \%$ after $30 \mathrm{kN}$ (Figure 6 (b)).

Figure 7 shows the progressive damage images along with the load displacement curve for a W-3 sample. A raised longitudinal crack occurred first from the edge of the hole and propagated along the 
binder tow which passed through the edge of the hole. Since this binder tow was at the edge of the hole, it passed through the stress concentration zone, which resulted in more straightening effect in this tow. Therefore it caused debonding and matrix cracking at the boundary of the tow at the surface. As the load increased, transverse cracks initiated mostly, but not exclusively, in between two weft tows since W-3 does not have distinctive resin rich channels in between every two weft tows as is the case with $\mathrm{W}-1$. In $\mathrm{W}-3$, the resin rich regions spread from the binding points along the weft tow for a very limited distance, and also there were fewer surface binding points in W-3 than in W-1. More debonding occurred as the transverse cracks propagated, as can be seen by the increase in the raised region, which led to the warp tow taking more load and finally fracturing. Since the transverse cracks did not always occur in between weft tows, some of the weft tows split along the length.

\subsection{Open-hole fatigue tests}

\subsubsection{TSA results}

During cyclic loading, the damage growth can cause local temperature rise which can be used as an indicator for damage progression [15, 26, 27]. The open-hole CFRP laminate sample studied in [26] showed high temperature concentration around the hole and also showed a temperature increase of over $10^{\circ} \mathrm{C}$ due to internal friction from delaminated layers. The temperature of $2 \mathrm{D}$ woven laminates increased during fatigue loading and also exhibited a "hot-zone" in the damaged area $[15,27]$. The sample temperature only varied for about $3^{\circ} \mathrm{C}$ in this study and the variation also followed the change in room temperature. Therefore the temperature change between cycles was not used as an indicator of damage, instead the processed $\Delta T$ distribution between the peak and valley of a cycle was used to indicate fatigue damage. The magnitude of $\Delta T(|\Delta T|)$ distribution was plotted in this paper since it can better highlight the damage feature. Since the binder tow and the matrix have different thermal expansion coefficients and were under different stress levels, the $|\Delta T|$ distribution also reflected the surface weave patterns as shown in Figure 10, Figure 12 (a), and Figure 13 (a). Similar phenomena have been observed in 2D textile composites, which shows about $0.2^{\circ} \mathrm{C}$ difference between the $\Delta T$ in the resin regions and in the tow regions [17, 23]. In order to distinguish and highlight the damage from the weave pattern, a $d \Delta T$ distribution map was obtained by subtracting the first $\Delta T$ map from all the other $\Delta T$ maps, which then highlighted all of the damage that occurred after the first recording.

Figure 8 shows the damage that occurred between 500 and 122,500 cycles, between 500 and 230,500 cycles, between 500 and 446,500, and between 524,500 and 992,500 cycles. As can be see, most of the damage initiated within the first 122,500 cycles and propagated at a lower speed afterwards. Only limited amount of damage occurred between 524,500 and 992,500 cycles, which indicated the damage propagation and new damage initiation process had slowed down. Figure 9 shows the $d \Delta T$ distribution of a W-3 sample during 1,000,000 cycles. Longitudinal cracks appeared at the very beginning of loading and did not propagate through-out the entire cyclic loading process. Transverse cracks initiated from the longitudinal cracks around the notch and propagated in the weft direction. Both in-tow and between-tow matrix cracks occurred within 124,500 cycles, and one between-tow matrix crack (crack-5 in Figure 10) appeared after 196,500 cycles from the longitudinal crack about $13 \mathrm{~mm}$ away from the notch. In order to see the damage propagation more clearly, the length of the cracks were measured on the $|\Delta T|$ maps as plotted in Figure 10. The crack propagation 
clearly showed two stages: initial propagation and stabilisation. Most of the cracks except crack-5 propagated more rapidly within 100,000 cycles after initiation and stabilised after 200,000 cycles. Crack-5 initiated at about 150,000 cycles and propagated for about 300,000 cycles before slowing down.

The damage initiation and progression scheme was similar in the other samples with different loading cycles. The surface damage area from the $d \Delta T$ maps was extracted and the percentage of damage area over the entire sample area was obtained at each recording. Figure 11 shows the surface damage area development during fatigue loading for each sample. As can be seen, the damage growth was the fastest in the first 100,000 cycles in both weaves and gradually slowed down afterwards. Although in the first 100,000 cycles the damage area percentage of both weaves were similar, the damage area increased at a slower rate in W-3 than in W-1 after 100,000 cycles. The damage area of W-3 almost saturated after 200,000 cycles while it kept increasing in the W-1 samples. Overall, the damage area was larger in W-1 samples than in W-3 samples because W-1 had more surface binding points that induced more matrix cracking than W-3. It should be noted that the damage area percentage only indicated the surface damage not the internal damage, because the thermal camera can only detect the temperature on the sample surface.

\subsubsection{Failure analysis}

The black paint was removed from the samples after testing for visual surface inspection, and microscopic photos were taken to examine the detail of the damage. The matrix cracks on the $\mathrm{W}-1$ samples were clearly observed. Figure 6 and 7 show the surface of W-1 and W-3 samples after $1,000,000$ cycles of fatigue loading with the last $|\Delta T|$ distribution maps.

In $\mathrm{W}-1$, matrix cracks initiated from the notch within the resin rich channels, and a few matrix cracks occurred at the binding points along the longitudinal crack as shown in the red ellipse in Figure 6 (a) and (b). Clear warp tow debonding can be seen from the microscopic image, which featured a light grey debonded interface through the resin rich channel, and was also found in the longitudinal direction along the edge of the notch. In addition, debonding also occurred to a small portion of the binder and weft tows in this region due to the surface matrix crack propagation. The warp tow debonding only existed in the region that was under tension. If a warp tow was partially under tension and partially broken due to the notch, the debonding interface would only exist in the tensioned part. As shown in Figure 12 (b) and (c), the boundary of the tensioning region is highlighted in red dash line which is tangential to the notch. The left part of the warp tow was cut off by the notch, therefore the warp tow interface remained intact showing black carbon. The right half of the warp tow passed through the stress concentration region and was straightened, therefore exhibited debonded interface (light grey). This tensioning difference also caused warp tow split, which were observed through the resin rich channel further away from the notch. The split region also showed a debonding area which was smaller in the sample with lower fatigue cycles.

In all W-3 samples, the cracks were not clearly seen from the surface of the sample because there were no large transparent resin regions like W-1 and the cracks in W-3 did not occur within the small resin rich regions. Most of the transverse cracks detected by the thermal camera were not visually observed on the surface especially the in-tow cracks, as can be seen in Figure 13 (a) and (b), while the longitudinal cracks were more pronounced but not as obvious as in $\mathrm{W}-1$, as shown in Figure 7 
(c). Both longitudinal and transverse cracks were more obvious after 1,000,000 cycles. As shown in Figure 13 (d), the transverse cracks (crack-2 in Figure 10) initiated from the tensioning boundary, propagated along the weft tow and into the depth of the specimen.

\subsubsection{Residual strength}

Since none of the samples were completely fractured after fatigue loading, further quasi-static tensile tests were conducted to obtain the residual tensile strength following the same testing procedure as the undamaged static tests. One W-3 sample was sectioned after 5,000,000 cycles to inspect the internal damage state, therefore were not tested for residual strength. The residual strength results are plotted in Figure 14 along with the averaged open-hole tensile strength. As can be seen, there is no clear correlation between the residual strength and fatigue cycles in W-1 and the residual strength of W-3 seems to increase with the fatigue cycles. The increase in residual strength due to fatigue damage has been previously found on open-hole carbon/epoxy laminates which showed about $20 \%$ improvement over 1,000,000 cycles [28]. It was reported that the fatigue damage altered the load path in the $0^{\circ}$ layer and redistributed the stress more evenly near the notch hence increased the residual strength [28]. However, the improvement in the W-3 samples was less than $10 \%$ over $1,000,000$ cycles and only five samples were tested. Therefore the correlation between the residual strength and fatigue cycles in W-3 is inconclusive. The only conclusion that can be drawn from this data is that the fatigue loading at this level does not dramatically affect the residual tensile strength, since the difference between the residual strength and the averaged open-hole tensile strength is within $10 \%$ in all W-1 and W-3 samples. Since the main fatigue damage only occurred to the matrix and no damage was observed on the load-carrying warp tow except fibre splitting near the notch, it is reasonable that the post-fatigue residual strength did not degrade from the tensile strength.

Overall, the two types of 3D woven composites showed no complete fracture after cyclic loading but only exhibited four types of damage: surface matrix cracking, warp tow splitting, warp tow debonding, and inter-weft-tow matrix cracking. Mouritz and Cox [2] reviewed that the 3D woven composite exhibited lower un-notched fatigue strength than the $2 \mathrm{D}$ equivalent laminate. A S-N curve suggested that the un-notched 2D laminate failed within 100,000 cycles at $60 \%$ of its static strength [2]. The open-hole tension-tension fatigue tests on a 2D pre-preg carbon/epoxy laminate [26] also showed final fracture around 100,000 cycles at $60 \%$ of its static strength. None of the 3D woven samples in this study fractured completely during fatigue loading even after 5,000,000 cycles at about $65 \%$ of their static strength.

\section{Conclusions}

The open-hole quasi-static tensile behaviour and tensile-tensile fatigue behaviour of two weaves W-1 and W-3 were mechanically characterised. The strain development during static tests was obtained using a DIC system and the damage propagation during fatigue loading was captured by a thermal camera. The results and findings from these tests are concluded as follows.

The open-hole quasi-static tensile tests revealed that W-3 exhibited less reduction in its tensile strength (1-9\%) than W-1 (17-20\%), and using a larger notch also had little effect on the tensile strength (less than 10\%), which was similar to the findings in the literature [10,11]. Digital image 
correlation maps revealed that the strain distribution of W-1 was more dependent on the weave pattern than W-3 due to its more pronounced surface resin channels.

The fatigue damage initiation and propagation were characterised through a set of tensile-tensile fatigue open-hole tests using a thermal camera. In both weaves, most of the damage initiated and propagated relatively rapidly within the first 100,000 cycles and slowed down afterwards. Based on the temperature gradient distribution maps, W-1 showed more damage sites and more rapid damage growth than W-3. The post-fatigue residual strength of both W-1 and W-3 samples showed less than

$10 \%$ difference from the averaged open-hole tensile strength. Due to the complexity and variety of $3 \mathrm{D}$ woven composites, it is rather difficult for the current testing standard to accommodate each type of $3 \mathrm{D}$ woven composite. Further investigation in the testing standard for 3D woven composites can be beneficial. More samples need to be tested to draw a conclusive correlation between the residual strength and fatigue loading, and higher fatigue load levels need to be applied to achieve final fatigue failure. No complete breakage occurred to any of the samples even after 5,000,000 cycles at about $65 \%$ of the static strength, while previous research in the literature showed a 2D laminate failed within 100,000 cycles at $60 \%$ of its static strength [2, 26].

Overall, W-3 showed higher open-hole static strength and exhibited fewer damage sites during tension-tension fatigue open-hole tests than $\mathrm{W}-1$. The confidence of fatigue properties can be improved with more specimens and ultimate fatigue failure can be achieved using higher stress levels.

\section{Acknowledgements}

This research project is supported by the Aeronautical and Automotive Engineering Department of Loughborough University and M.Wright \& Sons Ltd. The help and support of the department staff and M.Wright \& Sons Ltd are gratefully acknowledged.

\section{References}

[1] A. P. Mouritz, 6 - Three-dimensional (3D) fibre reinforcements for composites, Composite Reinforcements for Optimum Performance, Woodhead Publishing, 2011, pp. 157-199.

[2] A. P. Mouritz, B. N. Cox, A mechanistic interpretation of the comparative in-plane mechanical properties of 3D woven, stitched and pinned composites, Composites Part A: Applied Science and Manufacturing 41 (6) (2010) 709-728.

[3] J. N. Baucom, M. A. Zikry, Low-velocity impact damage progression in woven e-glass composite systems, Composites Part A: Applied Science and Manufacturing 36 (5) (2005) 658-664.

[4] F. Chen, J. M. Hodgkinson, Impact behaviour of composites with different fibre architecture, Proceedings of the Institution of Mechanical Engineers, Part G: Journal of Aerospace Engineering 223 (7) (2009) 1009-1017.

[5] D. S. Ivanov, S. V. Lomov, A. E. Bogdanovich, M. Karahan, I. Verpoest, A comparative study of tensile properties of non-crimp 3D orthogonal weave and multi-layer plain weave e-glass composites. Part II: Comprehensive experimental results, Composites Part A: Applied Science and Manufacturing 40 (8) (2009) 1144-1157. 
[6] S. Rudov-Clark, A. P. Mouritz, Tensile fatigue properties of a 3D orthogonal woven composite, Composites Part A: Applied Science and Manufacturing 39 (6) (2008) 1018-1024.

[7] Y. Q. Ding, Y. Yan, R. McIlhagger, D. Brown, Comparison of the fatigue behaviour of 2D and 3D woven fabric reinforced composites, Journal of Materials Processing Technology 55 (3-4) (1995) 171-177.

[8] A. P. Mouritz, Tensile fatigue properties of 3D composites with through-thickness reinforcement, Composites Science and Technology 68 (12) (2008) 2503-2510.

[9] M. Karahan, S. V. Lomov, A. E. Bogdanovich, I. Verpoest, Fatigue tensile behavior of carbon/epoxy composite reinforced with non-crimp 3D orthogonal woven fabric, Composites Science and Technology 71 (16) (2011) 1961-1972.

[10] K. H. Tsai, C. H. Chiu, T. H. Wu, Fatigue behavior of 3D multi-layer angle interlock woven composite plates, Composites Science and Technology 60 (2) (2000) 241-248.

[11] R. Muñoz, V. Martínez, F. Sket, C. González, J. LLorca, Mechanical behavior and failure micromechanisms of hybrid 3D woven composites in tension, Composites Part A: Applied Science and Manufacturing 59 (0) (2014) 93-104.

[12] B. N. Cox, M. S. Dadkhah, W. L. Morris, On the tensile failure of 3D woven composites, Composites Part A: Applied Science and Manufacturing 27 (6) (1996) 447-458.

[13] G. L. Rosa, C. Clienti, F. L. Savio, Fatigue analysis by acoustic emission and thermographic techniques, Procedia Engineering 74 (0) (2014) 261-268.

[14] J. Cuadra, P. A. Vanniamparambil, K. Hazeli, I. Bartoli, A. Kontsos, Damage quantification in polymer composites using a hybrid ndt approach, Composites Science and Technology 83 (0) (2013) 11-21.

[15] D. S. de Vasconcellos, F. Touchard, L. Chocinski-Arnault, Tension-tension fatigue behaviour of woven hemp fibre reinforced epoxy composite: A multi-instrumented damage analysis, International Journal of Fatigue 59 (0) (2014) 159-169.

[16] W. R. Broughton, M. R. L. Gower, M. J. Lodeiro, G. D. Pilkington, R. M. Shaw, An experimental assessment of open-hole tension-tension fatigue behaviour of a GFRP laminate, Composites Part A: Applied Science and Manufacturing 42 (10) (2011) 1310-1320.

[17] R. K. Fruehmann, J. M. Dulieu-Barton, S. Quinn, Assessment of fatigue damage evolution in woven composite materials using infra-red techniques, Composites Science and Technology 70 (6) (2010) 937-946.

[18] S. Dai, P. R. Cunningham, S. Marshall, C. Silva, Influence of fibre architecture on the tensile, compressive and flexural behaviour of 3d woven composites, Composites Part A: Applied Science and Manufacturing 69 (0) (2015) 195-207.

[19] University of Nottingham, Texgen (8 August 2013). 
[20] A. International, ASTM D5766 standard test method for open-hole tensile strength of polymer matrix composite laminates (2011).

[21] P. Stanley, W. K. Chan, The application of thermoelastic stress analysis techniques to composite materials, The Journal of Strain Analysis for Engineering Design 23 (3) (1988) 137-143.

[22] J. M. Dulieu-Barton, Introduction to thermoelastic stress analysis, Strain 35 (2) (1999) 35-39.

[23] R. K. Fruehmann, J. M. Dulieu-Barton, S. Quinn, On the thermoelastic response of woven composite materials, The Journal of Strain Analysis for Engineering Design 43 (6) (2008) $435-450$.

[24] A. F. Robinson, J. M. Dulieu-Barton, S. Quinn, R. L. Burguete, Paint coating characterization for thermoelastic stress analysis of metallic materials, Measurement Science and Technology 21 (8) (2010) 085502.

[25] J. P. Quinn, A. T. McIlhagger, R. McIlhagger, Examination of the failure of 3D woven composites, Composites Part A: Applied Science and Manufacturing 39 (2) (2008) 273-283.

[26] O. J. Nixon-Pearson, S. R. Hallett, P. J. Withers, J. Rouse, Damage development in open-hole composite specimens in fatigue. Part 1: experimental investigation, Composite Structures 106 (0) (2013) 882-889.

[27] L. Toubal, M. Karama, B. Lorrain, Damage evolution and infrared thermography in woven composite laminates under fatigue loading, International Journal of Fatigue 28 (12) (2006) $1867-1872$.

[28] R. Ambu, F. Aymerich, F. Bertolino, Investigation of the effect of damage on the strength of notched composite laminates by digital image correlation, The Journal of Strain Analysis for Engineering Design 40 (5) (2005) 451-461. 

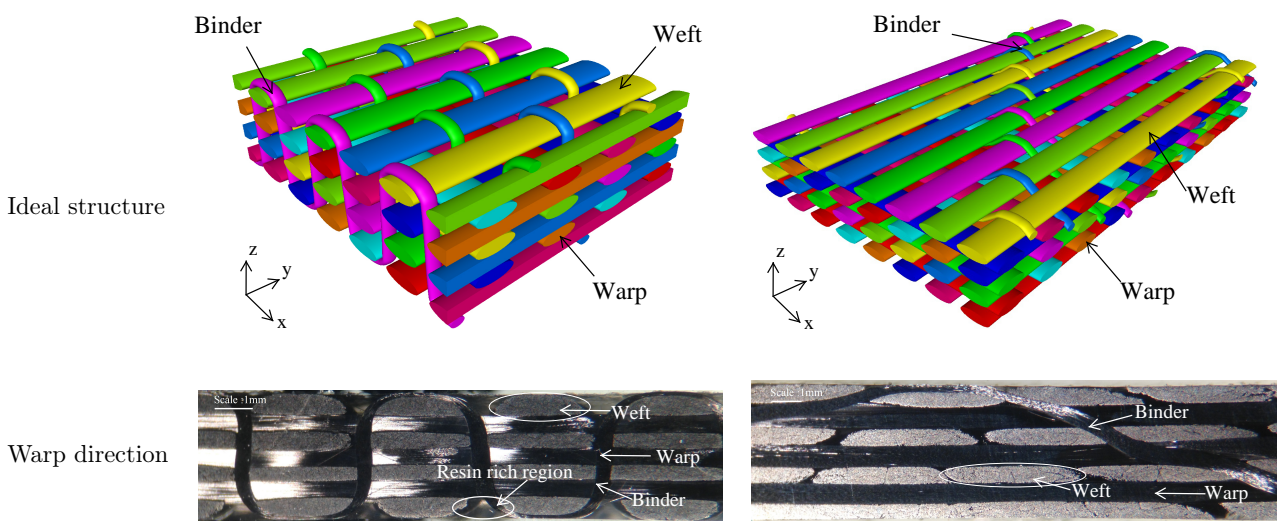

Top surface

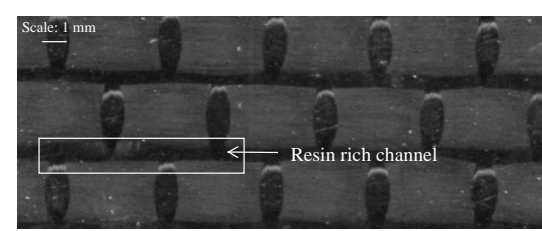

(a) W-1

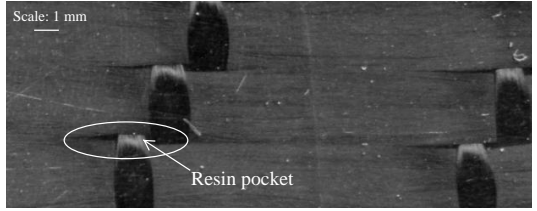

(b) W-3

Figure 1: Weave architectures 
(a) Surface

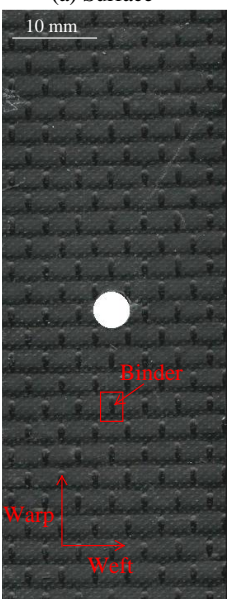

(b) $340 \mathrm{MPa}$

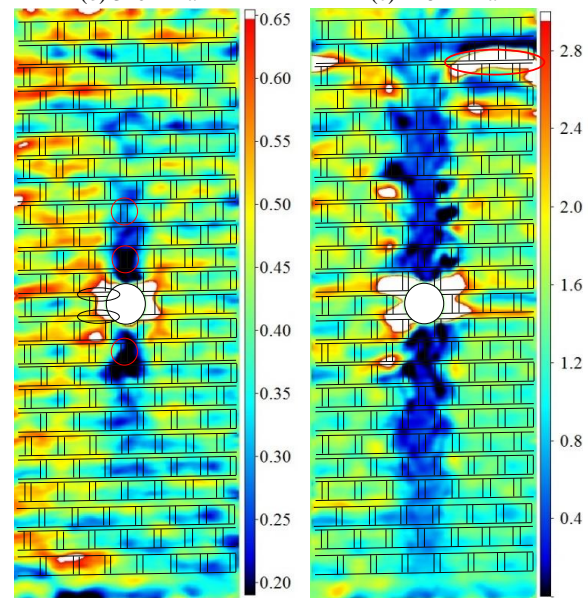

Figure 2: Longitudinal strain (\%) distribution of a standard W-1 sample at different net notch stress levels (DIC images).

(a) Surface

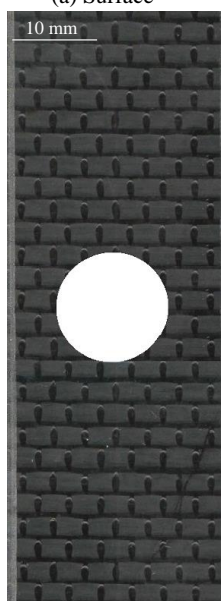

(b) $303 \mathrm{MPa}$

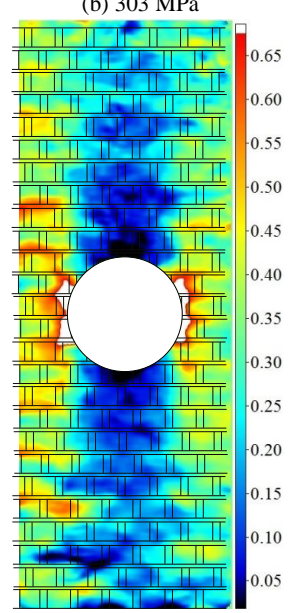

(c) $1116 \mathrm{MPa}$

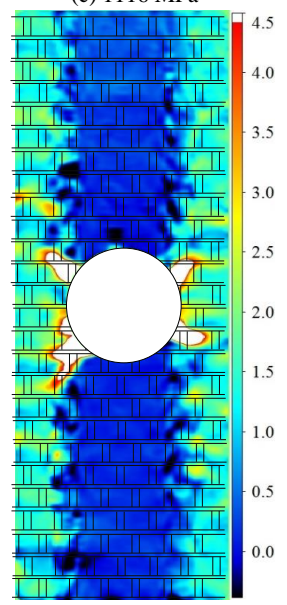

Figure 3: Longitudinal strain (\%) distribution of an enlarged W-1 sample at different net notch stress levels (DIC images). 
(a) Surface

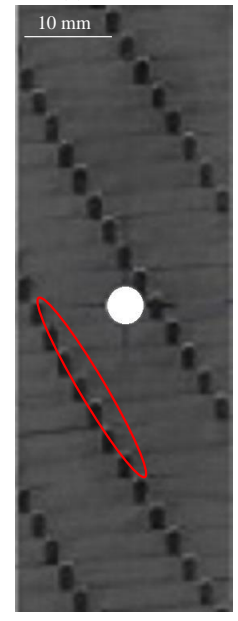

(b) $295 \mathrm{MPa}$

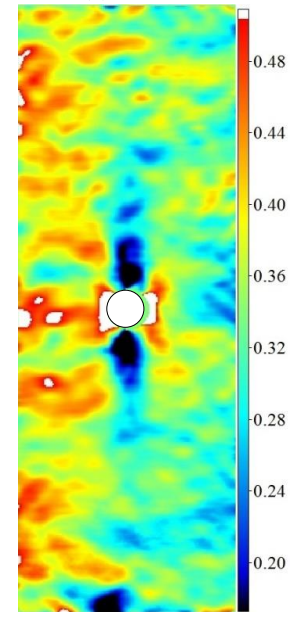

(c) $1258 \mathrm{MPa}$

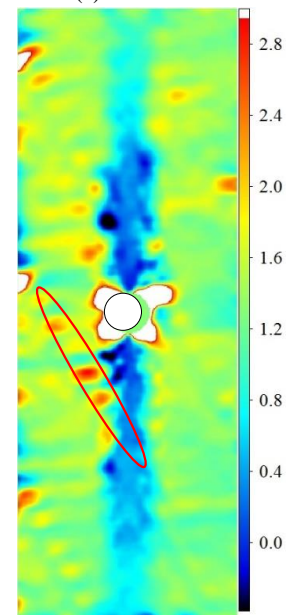

Figure 4: Longitudinal strain (\%) distribution of a standard W-3 sample at different net notch stress levels (DIC images).

(a) Surface

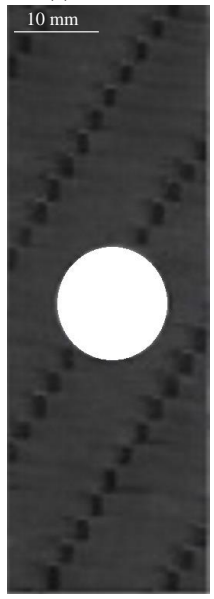

(b) $305 \mathrm{MPa}$

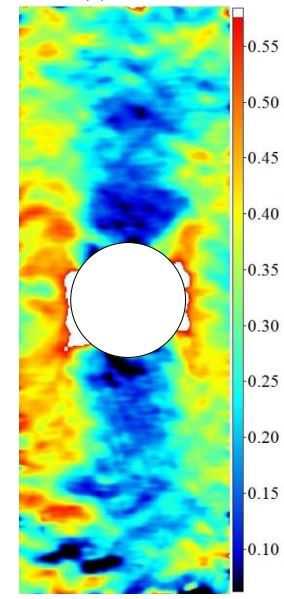

(c) $1189 \mathrm{MPa}$

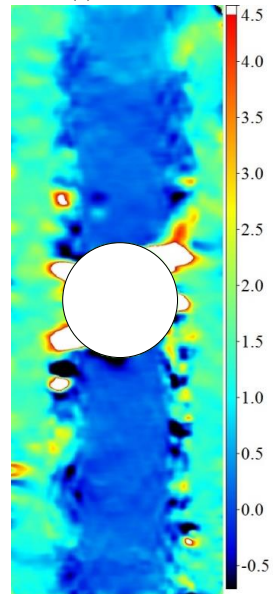

Figure 5: Longitudinal strain (\%) distribution of an enlarged W-1 sample at different net notch stress levels (DIC images). 


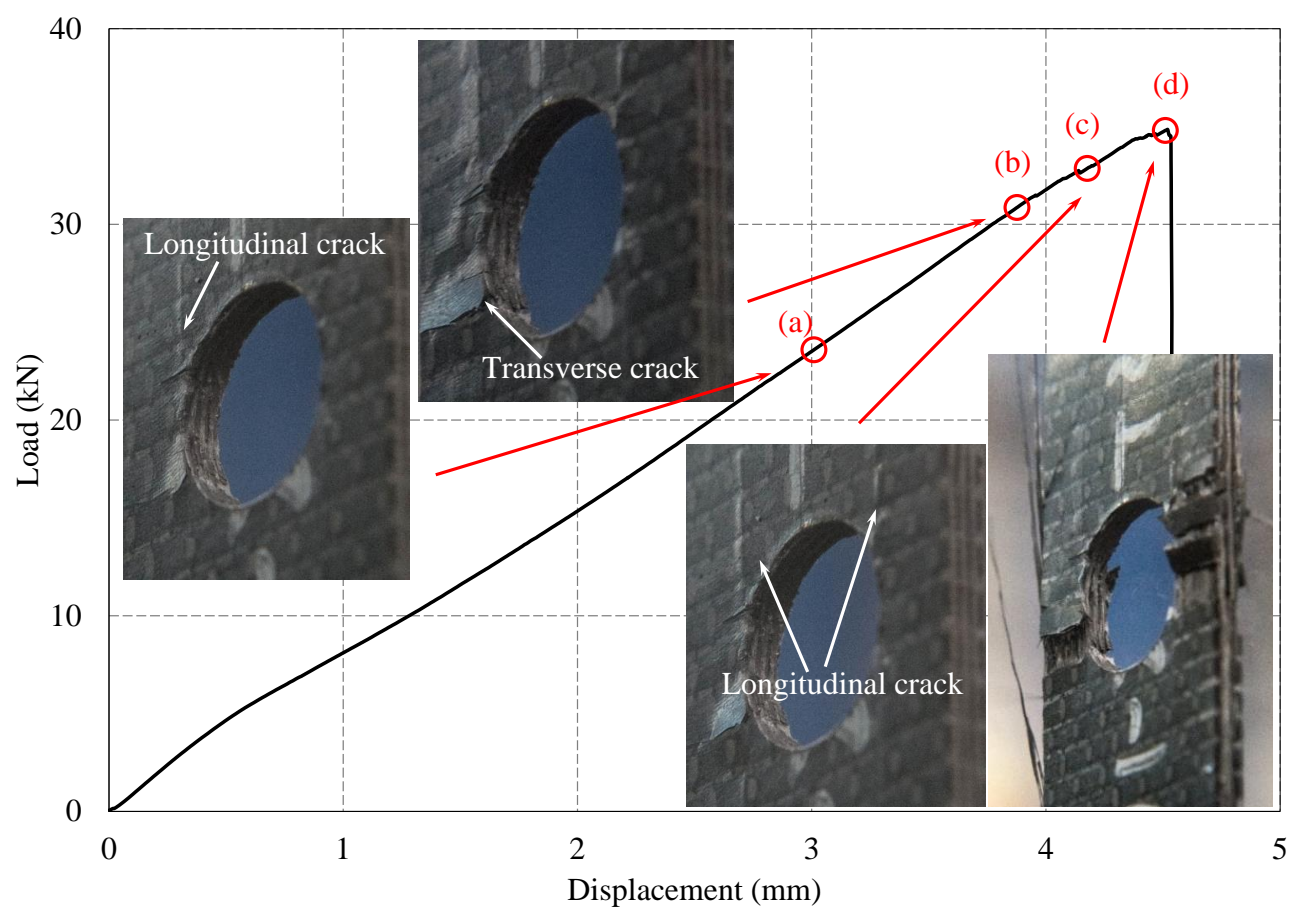

Figure 6: Load-displacement curve of a W-1 sample with damage images.

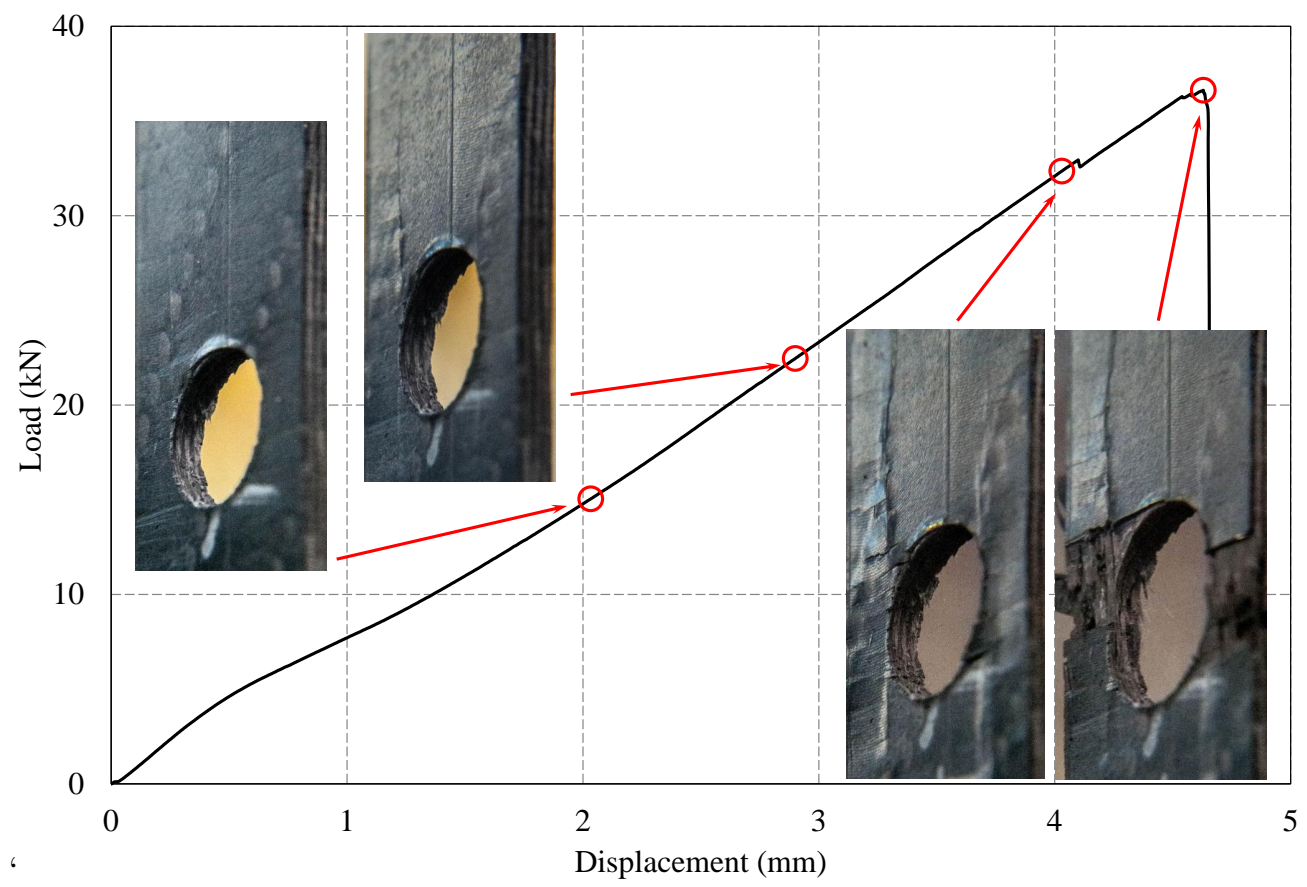

Figure 7: Load-displacement curve of a W-3 sample with damage images. 

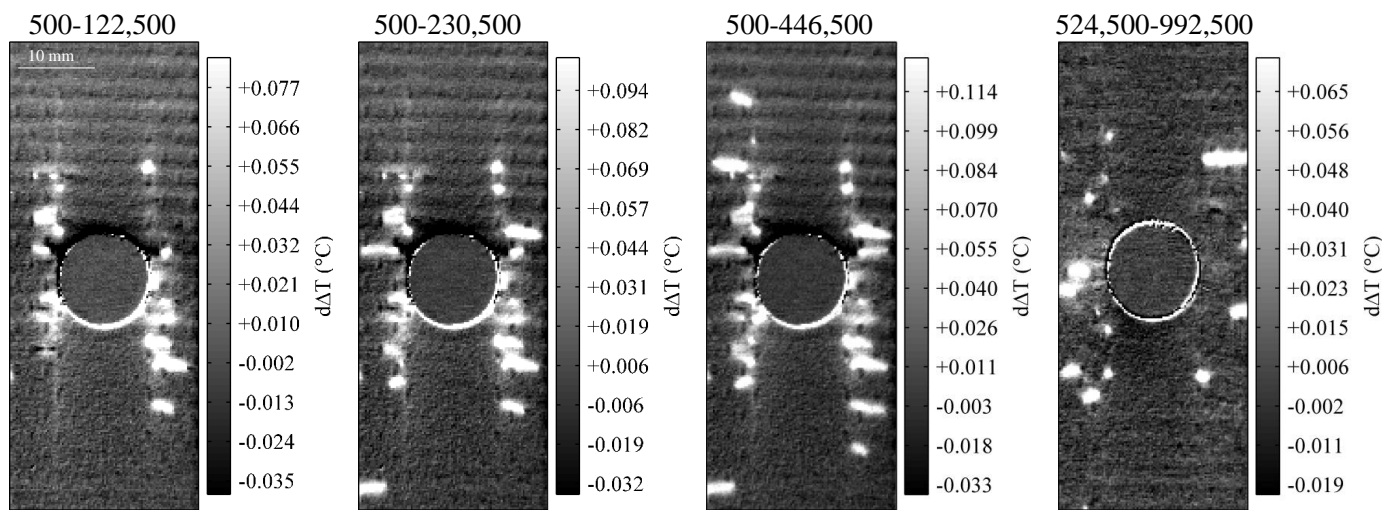

Figure 8: $d \Delta T$ distribution maps of a W-1 sample during 1,000,000 cycles.
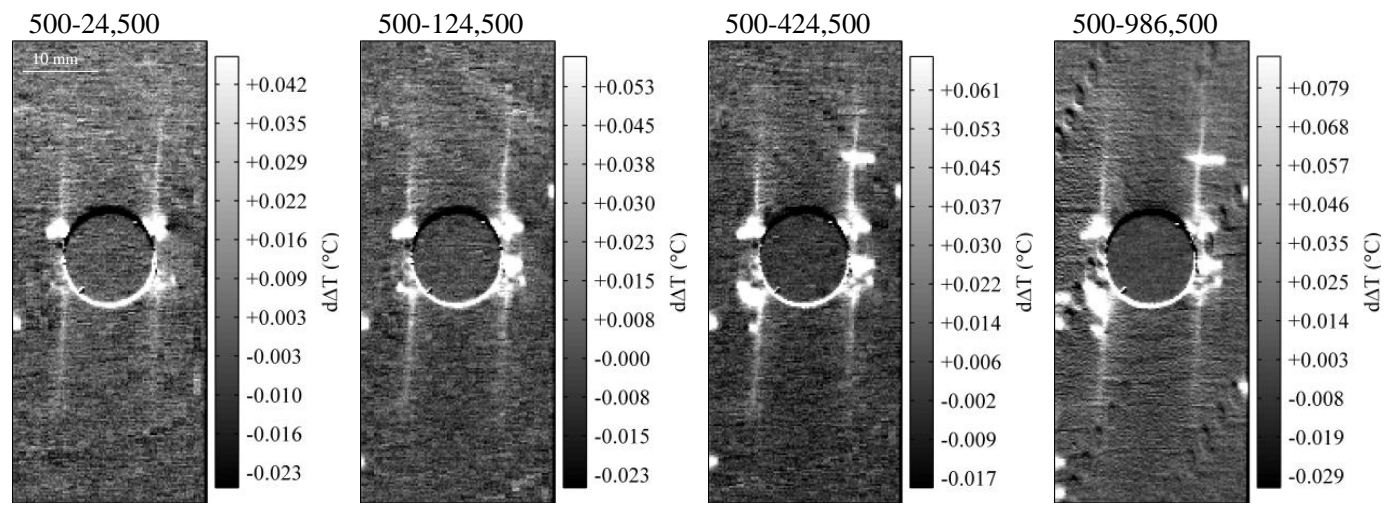

Figure 9: $d \Delta T$ distribution maps of a W-3 sample during 1,000,000 cycles.
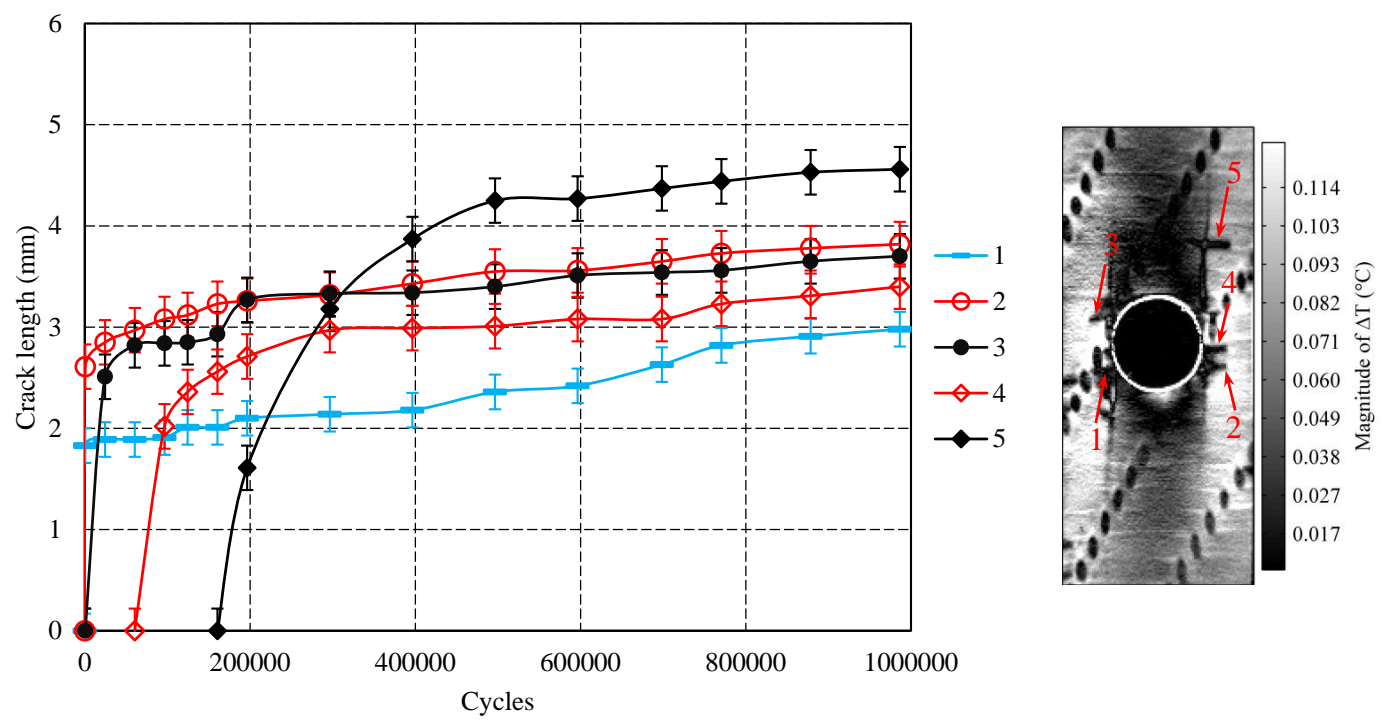

Figure 10: Cracks propagation of a W-3 sample. 


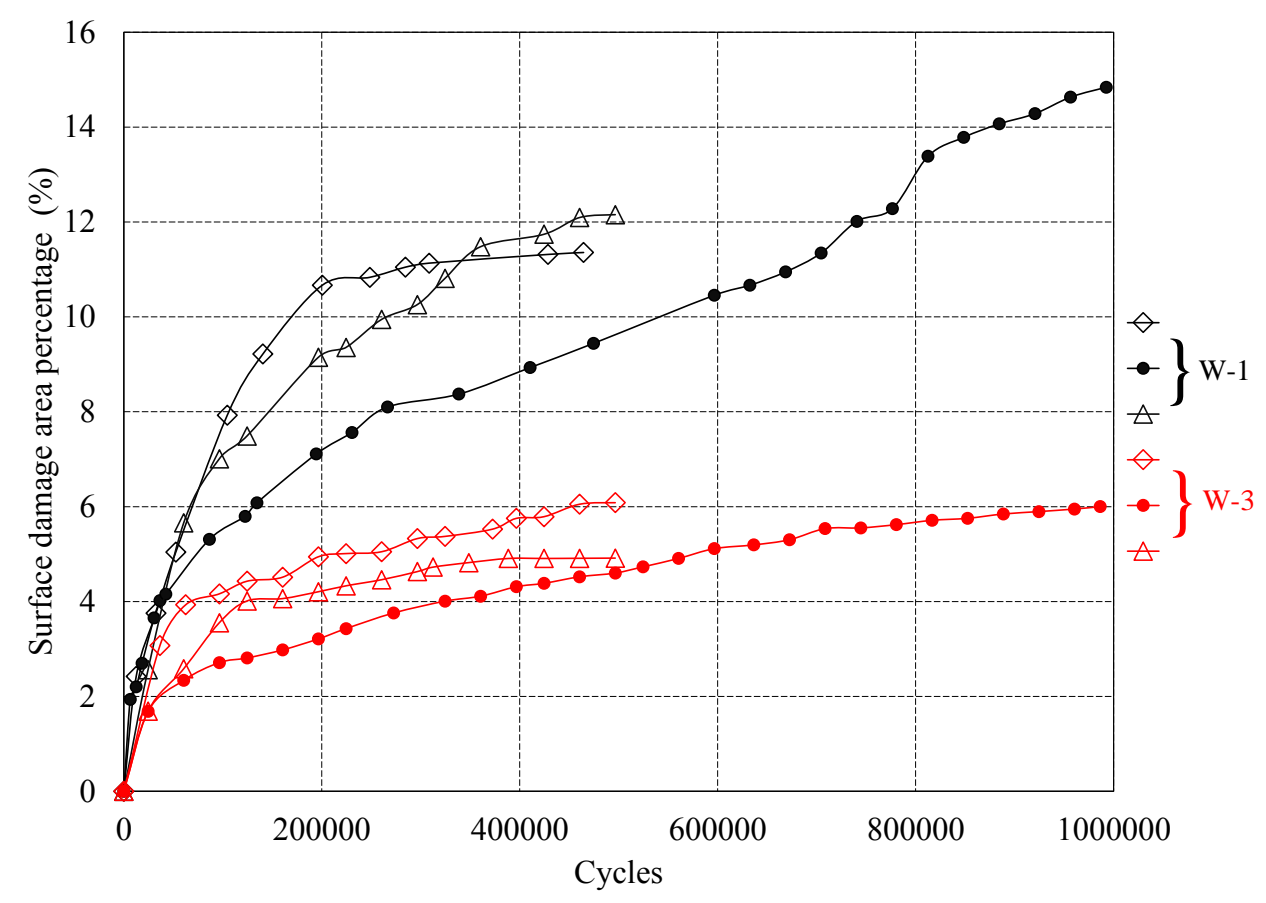

Figure 11: Damage area percentage during cyclic loading of W-1 and W-3 fatigue samples.

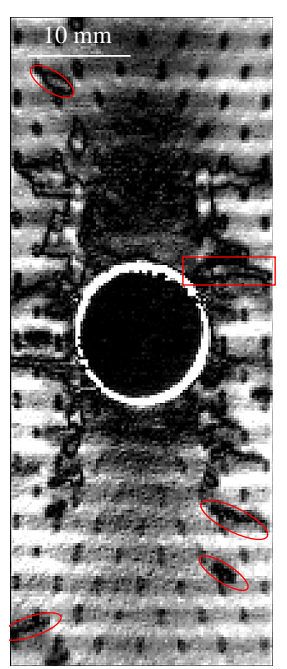

(a)

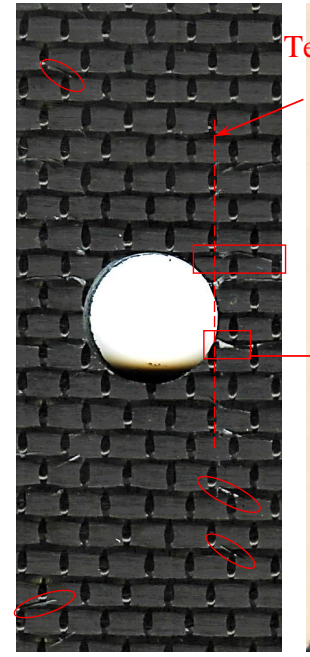

(b)

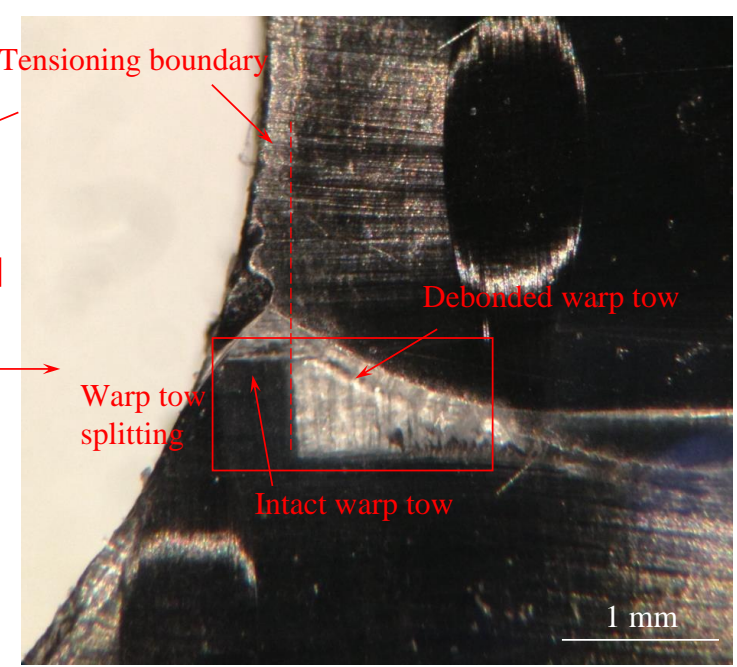

(c)

Figure 12: Fatigue damage of a W-1 sample after 1,000,000 cycles: (a) $|\Delta T|$ distribution showing surface damage pattern, (b) sample surface, (c) detailed local damage. 


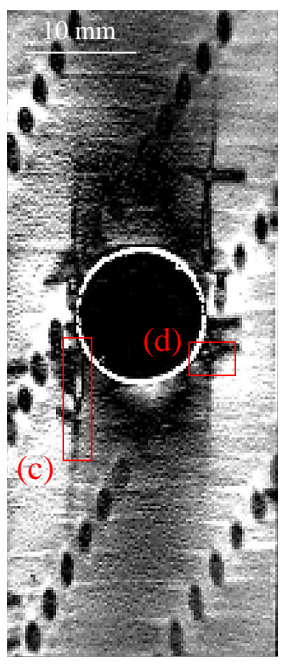

(a)

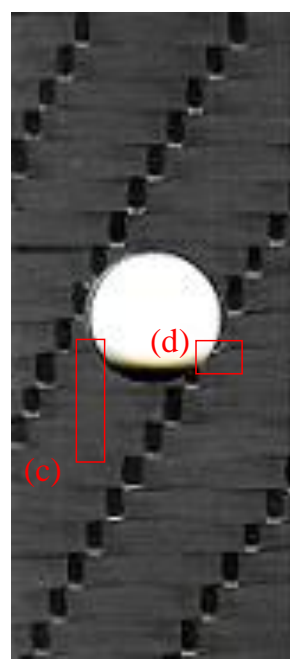

(b)

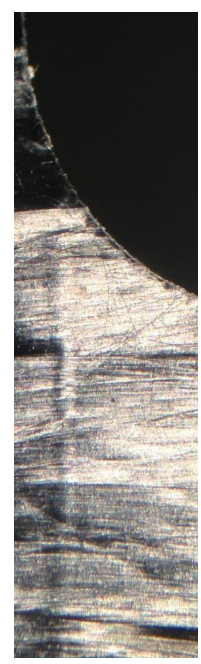

(c)

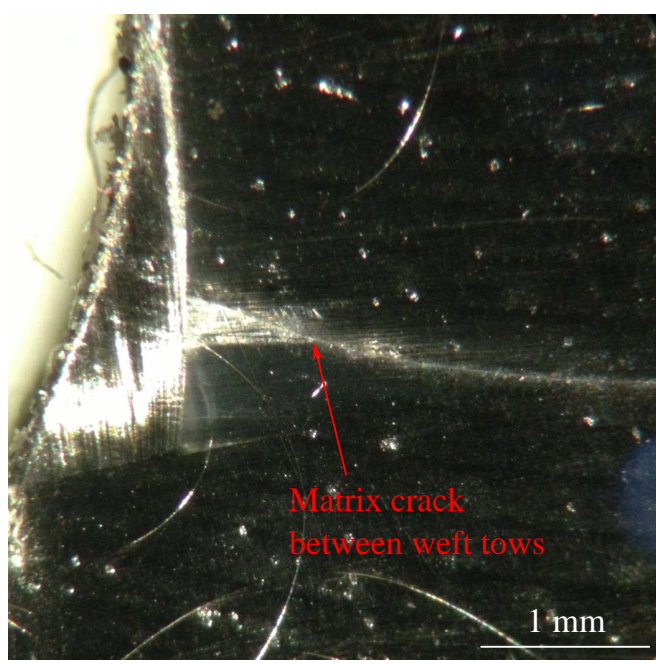

(d)

Figure 13: Fatigue damage of a W-3 sample after 1,000,000 cycles: (a) $|\Delta T|$ distribution showing surface damage pattern, (b) sample surface, (c) longitudinal damage, (d) transverse damage near the notch.

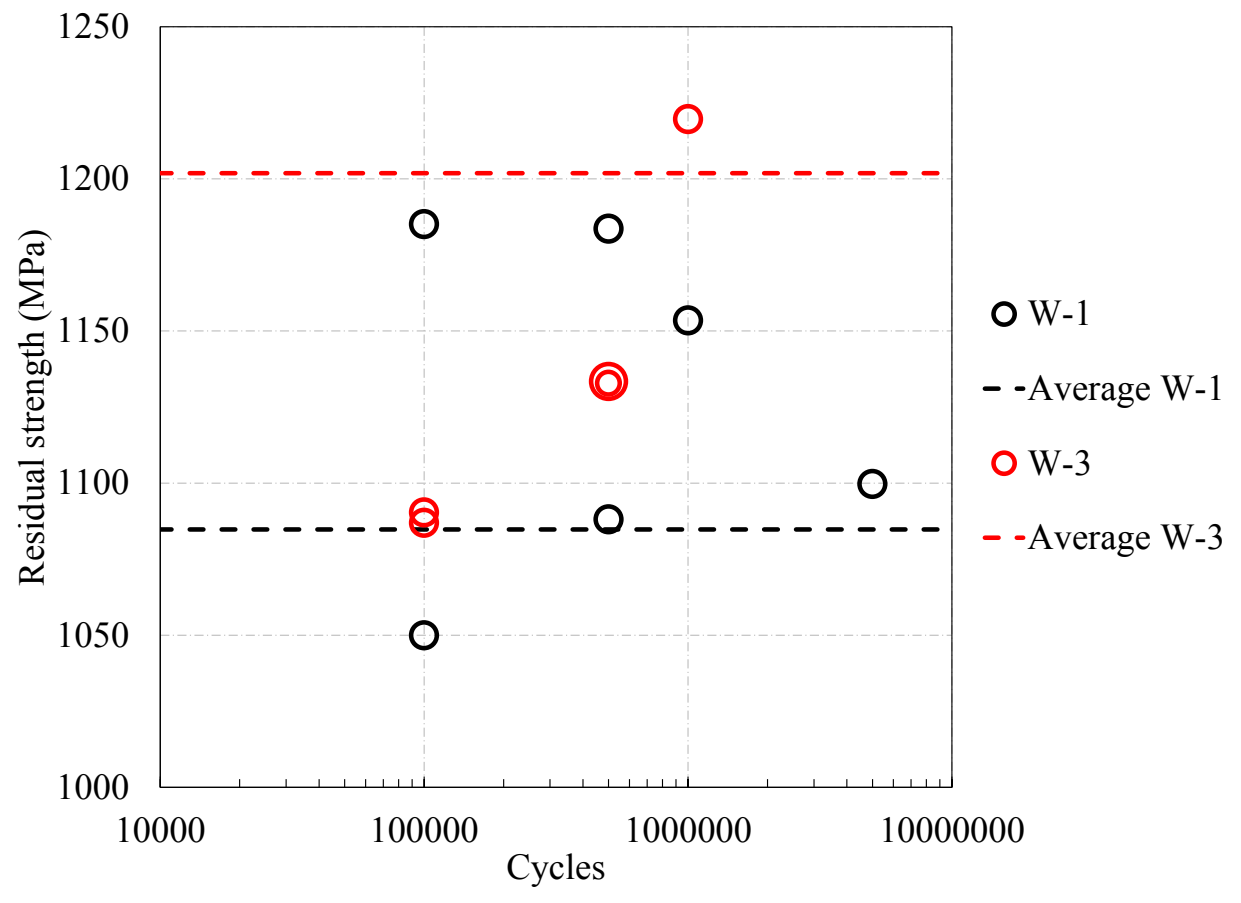

Figure 14: Post-fatigue residual tensile strength. 
Table 1: Averaged quasi-static open-hole tests results.

\begin{tabular}{cccccc}
\hline Weave & $\phi(\mathrm{mm})$ & Notched strength $(\mathrm{MPa})$ & CV $(\%)$ & Un-notched strength (MPa) & CV (\%) \\
\hline \multirow{2}{*}{$\mathrm{W}-1$} & 4.1 & 1145.71 & 4.50 & \multirow{2}{*}{1358.54} & \multirow{2}{*}{3.22} \\
& 12.5 & 1093.98 & 4.65 & & \multirow{2}{*}{5.84} \\
\hline \multirow{2}{*}{$\mathrm{W}-3$} & 4.1 & 1265.01 & 3.61 & \multirow{2}{*}{1280.99} & \\
& 12.5 & 1201.87 & 8.03 & & \\
\hline
\end{tabular}

